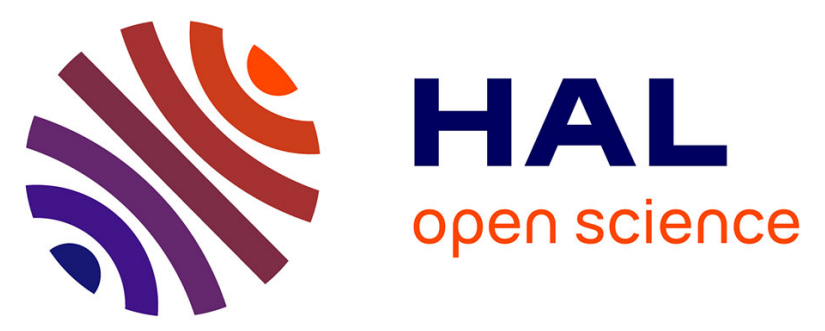

\title{
CONVERGENCE ANALYSIS OF TWO NUMERICAL SCHEMES APPLIED TO A NONLINEAR ELLIPTIC PROBLEM
}

Christine Bernardi, Jad Dakroub, Gihane Mansour, Farah Rafei, Toni Sayah

\section{- To cite this version:}

Christine Bernardi, Jad Dakroub, Gihane Mansour, Farah Rafei, Toni Sayah. CONVERGENCE ANALYSIS OF TWO NUMERICAL SCHEMES APPLIED TO A NONLINEAR ELLIPTIC PROBLEM. 2015. hal-01154534

\section{HAL Id: hal-01154534 https: / hal.sorbonne-universite.fr/hal-01154534}

Preprint submitted on 22 May 2015

HAL is a multi-disciplinary open access archive for the deposit and dissemination of scientific research documents, whether they are published or not. The documents may come from teaching and research institutions in France or abroad, or from public or private research centers.
L'archive ouverte pluridisciplinaire HAL, est destinée au dépôt et à la diffusion de documents scientifiques de niveau recherche, publiés ou non, émanant des établissements d'enseignement et de recherche français ou étrangers, des laboratoires publics ou privés. 


\title{
CONVERGENCE ANALYSIS OF TWO NUMERICAL SCHEMES APPLIED TO A NONLINEAR ELLIPTIC PROBLEM
}

\author{
CHRISTINE BERNARDI ${ }^{\dagger}$, JAD DAKROUB $^{\dagger \ddagger}$, GIHANE MANSOUR $^{\ddagger}$, FARAH RAFEI $^{\ddagger}$ AND TONI SAYAH ${ }^{\ddagger}$.
}

\begin{abstract}
For a given nonlinear problem discretized by standard finite elements, we propose two iterative schemes to solve the discrete problem. We prove the well-posedness of the corresponding problems and their convergence. Next, we construct error indicators and prove optimal a posteriori estimates where we treat separately the discretization and linearization errors. Some numerical experiments confirm the validity of the schemes and allow us to compare them.
\end{abstract}

\section{INTRODUCTION}

Let $\Omega$ be an open polygon in $\mathbb{R}^{2}$, we consider the problem

$$
\begin{aligned}
-\Delta u+\lambda|u|^{2 p} u & =f \quad \text { in } \quad \Omega, \\
u & =0 \quad \text { on } \partial \Omega,
\end{aligned}
$$

where $\lambda$ and $p$ are two positive real numbers. The right-hand side $f$ belongs to the dual space $H^{-1}(\Omega)$ of the Sobolev space $H_{0}^{1}(\Omega)$. The a posteriori error analysis of finite element approximations of the present model problem has been studied by Bernardi, Dakroub, Mansour and Sayah, see [2]. In fact, let $V_{h} \subset H_{0}^{1}(\Omega)$ be the $\mathcal{P}_{1}$ finite element space associated with a regular family of triangulations of $\Omega$, denoted by $\mathcal{T}_{h}$. Using $\mathcal{P}_{1}$ Lagrange finite elements, the discrete variational problem obtained by the Galerkin method amounts to (from now on, we denote by $(\cdot, \cdot)$ the scalar product of $L^{2}(\Omega)$ )

Find $u_{h} \in V_{h}$ such that

$$
\forall v_{h} \in V_{h}, \quad\left(\nabla u_{h}, \nabla v_{h}\right)+\lambda\left(\left|u_{h}\right|^{2 p} u_{h}, v_{h}\right)=\left\langle f, v_{h}\right\rangle .
$$

In order to solve the discrete nonlinear problem (1.3), we introduced in [2] the following linear numerical scheme, called fixed-point algorithm:

Find $u_{h}^{i+1} \in V_{h}$ such that

$$
\forall v_{h} \in V_{h}, \quad\left(\nabla u_{h}^{i+1}, \nabla v_{h}\right)+\lambda\left(\left|u_{h}^{i}\right|^{2 p} u_{h}^{i+1}, v_{h}\right)=\left\langle f, v_{h}\right\rangle .
$$

This algorithm leads to a conditional convergence of the problem. In fact, the convergence of this numerical schemes depends on the parameters $\lambda, p$ and $f$. Furthermore, the a priori estimate of the discrete variationel problem is presented in [2]. As well, the a posteriori analysis of the discretization is performed but requires that the discrete solution belongs to a neighborhood of the exact solution $u$.

As a new contribution to the previous work that we have carried out recently on the a posteriori analysis of the present nonlinear problem, see [2], we introduce in this paper two different convergent numerical schemes to solve this problem. In fact, the main idea is to introduce a parameter $\alpha$ which can be controlled in order to insure the convergence. Let $u_{h}^{0}$ be an initial guess, for $i \geq 0$ we introduce the following two algorithms:

May 22, 2015

$\dagger$ CNRS, UMR 7598, Laboratoire Jacques-Louis Lions, F-75005, Paris, France.

Sorbonne Universits, UPMC Univ Paris 06, UMR 7598, LJLI, F-75005, Paris, France.

¥ Unité de recherche EGFEM, Faculté des sciences, Université Saint-Joseph, Lebanon.

bernardi@ann.jussieu.fr, jad.dakroub@usj.edu.lb, gihane.mansour@usj.edu.lb, farah.rafei@net.usj.edu.lb, toni.sayah@usj.edu.lb. 
First numerical scheme.

Find $u_{h}^{i+1} \in V_{h}$ such that

$$
\forall v_{h} \in V_{h}, \quad \alpha\left(u_{h}^{i+1}-u_{h}^{i}, v_{h}\right)+\left(\nabla u_{h}^{i+1}, \nabla v_{h}\right)+\lambda\left(\left|u_{h}^{i}\right|^{2 p} u_{h}^{i+1}, v_{h}\right)=\left\langle f, v_{h}\right\rangle,
$$

\section{Second numerical scheme.}

Find $u_{h}^{i+1} \in V_{h}$ such that

$$
\forall v_{h} \in V_{h}, \quad \alpha\left(\nabla u_{h}^{i+1}-\nabla u_{h}^{i}, \nabla v_{h}\right)+\left(\nabla u_{h}^{i+1}, \nabla v_{h}\right)+\lambda\left(\left|u_{h}^{i}\right|^{2 p} u_{h}^{i+1}, v_{h}\right)=\left\langle f, v_{h}\right\rangle,
$$

For a parameter $\alpha$ bigger than a specific constant that depends on $\lambda, p$ and the data $f$, problem (1.5) and (1.6) always converge. Moreover, our objective is to derive an a posteriori error estimate distinguishing linearization and discretization errors.

In practice, the present problem (1.1) - (1.2) is solved using an iterative method involving a linearization process and approximated by the finite element method. Thus, two sources of error appear, namely linearization and discretization. The main result in [2] is a two-sided bound of the error distinguishing linearization and discretization errors in the context of an adaptive procedure. This type of analysis was introduced by A.-L. Chaillou and M. Suri [3, 4] for a general class of problems characterized by strongly monotone operators and developed by L. El Alaoui, A. Ern and M. Vohralík [5] for a class of secondorder monotone quasi-linear diffusion-type problems approximated by piecewise affine, continuous finite elements. We wish to extend these results to the problem that we consider and prove optimal estimates.

The paper is organized as follows:

- Section 2 describes the model problem.

- Section 3 is devoted to the study of the convergence of the schemes.

- Section 4 provides the a posteriori estimates for both problems.

- Section 5 is devoted to the numerical results.

\section{Preliminaries}

In this section, we describe the variational formulation associated with the nonlinear problem (1.1)-(1.2) and introduce and recall some corresponding properties which will be used later.

We denote by $L^{p}(\Omega)$ the space of measurable functions summable with power $p$, and for all $v \in L^{p}(\Omega)$, the corresponding norm is defined by

$$
\|v\|_{L^{p}(\Omega)}=\left(\int_{\Omega}|v(\mathbf{x})|^{p} d \mathbf{x}\right)^{1 / p} .
$$

In the case $p=2$, we also denote this norm by $\|\cdot\|_{0, \Omega}$. Throughout this paper, we constantly use the classical Sobolev space

$$
H^{1}(\Omega)=\left\{v \in L^{2}(\Omega) ; \frac{\partial v}{\partial x_{1}}, \frac{\partial v}{\partial x_{2}} \in L^{2}(\Omega)\right\},
$$

which is equipped respectively with the semi-norm and norm

$$
\left.|v|_{1, \Omega}=\left(\int_{\Omega}\left(\left|\frac{\partial v}{\partial x_{1}}\right|^{2}+\left|\frac{\partial v}{\partial x_{2}}\right|^{2}\right) d \mathbf{x}\right)^{1 / 2} \quad \text { and } \quad\|v\|_{1, \Omega}=\left(\|v\|_{0, \Omega}^{2}+|v|_{1, \Omega}^{2}\right)\right)^{1 / 2} .
$$

In particular, we consider the following space

$$
H_{0}^{1}(\Omega)=\left\{v \in H^{1}(\Omega), v_{\mid \partial \Omega}=0\right\},
$$

and its dual space $H^{-1}(\Omega)$. We recall the Sobolev imbeddings (see Adams [1], Chapter 3).

Lemma 2.1. For any domain $\Omega$ in $\mathbb{R}^{2}$, for all $j, 1 \leq j<\infty$, there exists a positive constant $S_{j}$ such that

$$
\forall v \in H_{0}^{1}(\Omega), \quad\|v\|_{L^{j}(\Omega)} \leq S_{j}|v|_{1, \Omega}
$$


Remark 2.2. For domains $\Omega$ in $\mathbb{R}^{3}$, inequality (2.1) with standard definition of $H_{0}^{1}(\Omega)$ remains valid only for $j \leq 6$, whence the interest of working in dimension $d=2$.

Setting $X=H_{0}^{1}(\Omega)$, the model problem (1.1)-(1.2) admits the equivalent variational formulation:

Find $u \in X$ such that

$$
\forall v \in X, \quad \int_{\Omega} \nabla u \nabla v d \mathbf{x}+\int_{\Omega} \lambda|u|^{2 p} u v d \mathbf{x}=\langle f, v\rangle,
$$

Theorem 2.3. [2] Problem (2.2) admits a unique solution $u \in X$.

We now introduce the following technical lemmas:

Lemma 2.4. Let $a, b$ and $p$ be three real numbers. We have the following relation

$$
\left.|| a\right|^{p}-|b|^{p}|\leq p| a-b \mid\left(|a|^{p-1}+|b|^{p-1}\right) .
$$

Proof. The result follows from applying the mean value theorem to $f(x)=x^{p}$ with $x>0$.

Lemma 2.5. For all $x, y \in \mathbb{R}$ and $p \in \mathbb{R}$, we have

$$
\left(|x|^{2 p} x-|y|^{2 p} y\right)(x-y) \geq 0 .
$$

Remark 2.6. In the sequel, we denote by $C, C^{\prime}, \ldots$ generic constants that can vary from line to line but are always independent of all discretization parameters.

\section{Finite element discretization and Convergence}

In this section, we begin to collect some useful notation concerning the discrete setting and the a priori estimate. Then, we show the convergence of the schemes (1.5) and (1.6).

Let $\left(\mathcal{T}_{h}\right)_{h}$ be a regular family of triangulations of $\Omega$, in the sense that, for each $h$ :

- The union of all elements of $\mathcal{T}_{h}$ is equal to $\bar{\Omega}$.

- The intersection of two different elements of $\mathcal{T}_{h}$, if not empty, is a vertex or a whole edge of both triangles.

- The ratio of the diameter $h_{K}$ of any element $K$ of $\mathcal{T}_{h}$ to the diameter of its inscribed circle is smaller than a constant independent of $h$.

As usual, $h$ stands for the maximum of the diameters $h_{K}, K \in \mathcal{T}_{h}$. Let $V_{h} \subset H_{0}^{1}(\Omega)$ be the Lagrange $\mathcal{P}_{\ell}$ finite element space associated with $\mathcal{T}_{h}$, more precisely

$$
V_{h}=\left\{v_{h} \in H_{0}^{1}(\Omega) ; \forall K \in \mathcal{T}_{h}, v_{h_{\mid K}} \in \mathcal{P}_{\ell}(K)\right\},
$$

where $\mathcal{P}_{\ell}(K)$ stands for the space of restrictions to $K$ of polynomial functions of degree $\leq \ell$ on $\mathbb{R}^{2}$.

Remark 3.1. (Inverse inequality) There exists a constant $S_{I}>0$ such that for all $v_{h} \in V_{h}$ and $K \in \mathcal{T}_{h}$, we have

$$
\left|v_{h}\right|_{1, K} \leq S_{I} h_{K}^{-1}\left\|v_{h}\right\|_{0, K} .
$$

Theorem 3.2. [2] Let $u$ be the solution of (2.2). Then, Problem (1.3) has a unique solution $u_{h}$. Moreover, if $u \in H^{2}(\Omega)$, the followng estimate holds

$$
\left\|u_{h}-u\right\|_{1, \Omega} \leq C h\|u\|_{2, \Omega} .
$$

In the following, we investigate the convergence of the schemes (1.5) and (1.6).

Theorem 3.3. Problem (1.5) admits a unique solution. Furthermore, if the initial value $u_{h}^{0}$ satisfies the condition

$$
\left\|u_{h}^{0}\right\|_{0, \Omega} \leq S_{2}\|f\|_{-1, \Omega},
$$

then the solution of the problem (1.5) satisfies the estimates

$$
\left\|u_{h}^{i+1}\right\|_{0, \Omega} \leq S_{2}\|f\|_{-1, \Omega} \quad \text { and } \quad\left|u_{h}^{i+1}\right|_{1, \Omega} \leq \sqrt{1+\alpha S_{2}^{2}}\|f\|_{-1, \Omega} .
$$


Proof. It is readily checked that problem (1.5) has a unique solution as a consequence of the coercivity of the bilinear form.

We consider the equation (1.5) with $v_{h}=u_{h}^{i+1}$ and we obtain :

$$
\frac{\alpha}{2}\left\|u_{h}^{i+1}\right\|_{0, \Omega}^{2}-\frac{\alpha}{2}\left\|u_{h}^{i}\right\|_{0, \Omega}^{2}+\frac{\alpha}{2}\left\|u_{h}^{i+1}-u_{h}^{i}\right\|_{0, \Omega}^{2}+\left|u_{h}^{i+1}\right|_{1, \Omega}^{2}+\lambda\left(\left|u_{h}^{i}\right|^{2 p} u_{h}^{i+1}, u_{h}^{i+1}\right)=\left(f, u_{h}^{i+1}\right) .
$$

By using the inequality

$$
\left(f, u_{h}^{i+1}\right) \leq \frac{1}{2}\|f\|_{-1, \Omega}^{2}+\frac{1}{2}\left|u_{h}^{i+1}\right|_{1, \Omega}^{2},
$$

we deduce the relation

$$
\frac{\alpha}{2}\left\|u_{h}^{i+1}\right\|_{0, \Omega}^{2}-\frac{\alpha}{2}\left\|u_{h}^{i}\right\|_{0, \Omega}^{2}+\frac{\alpha}{2}\left\|u_{h}^{i+1}-u_{h}^{i}\right\|_{0, \Omega}^{2}+\frac{1}{2}\left|u_{h}^{i+1}\right|_{1, \Omega}^{2}+\lambda\left(\left|u_{h}^{i}\right|^{2 p} u_{h}^{i+1}, u_{h}^{i+1}\right) \leq \frac{1}{2}\|f\|_{-1, \Omega}^{2} .
$$

We now prove the first estimate in (3.3) by induction on $i$. Starting with the relation (3.2), we suppose that we have

$$
\left\|u_{h}^{i}\right\|_{0, \Omega} \leq S_{2}\|f\|_{-1, \Omega}
$$

We are in one of the following two situations :

- We have $\left\|u_{h}^{i+1}\right\|_{0, \Omega} \leq\left\|u_{h}^{i}\right\|_{0, \Omega}$. We obviously deduce the bound

$$
\left\|u_{h}^{i+1}\right\|_{0, \Omega} \leq S_{2}\|f\|_{-1, \Omega}
$$

from the induction hypothesis.

- We have $\left\|u_{h}^{i+1}\right\|_{0, \Omega} \geq\left\|u_{h}^{i}\right\|_{0, \Omega}$. The equation (3.4) gives

$$
\left|u_{h}^{i+1}\right|_{1, \Omega}^{2} \leq \|\left. f\right|_{-1, \Omega} ^{2}
$$

and we deduce the inequality

$$
\begin{aligned}
\left\|u_{h}^{i+1}\right\|_{0, \Omega}^{2} & \leq S_{2}^{2}\left|u_{h}^{i+1}\right|_{1, \Omega}^{2} \\
& \leq S_{2}^{2} \|\left. f\right|_{-1, \Omega} ^{2} .
\end{aligned}
$$

This gives the first part of (3.3). We now check the second part. We have from (3.4)

$$
\left|u_{h}^{i+1}\right|_{1, \Omega}^{2} \leq\|f\|_{-1, \Omega}^{2}+\alpha\left\|u_{h}^{i}\right\|_{0, \Omega}^{2} \leq\left(1+\alpha S_{2}^{2}\right)\|f\|_{-1, \Omega}^{2},
$$

whence the desired result.

Theorem 3.4. Problem (1.6) admits a unique solution. Furthermore, if the initial value $u_{h}^{0}$ verify the condition

$$
\left|u_{h}^{0}\right|_{1, \Omega} \leq \|\left. f\right|_{-1, \Omega},
$$

then the solution of Problem (1.6) satisfies the estimate

$$
\left|u_{h}^{i+1}\right|_{1, \Omega} \leq \|\left. f\right|_{-1, \Omega} .
$$

Proof. We follow the same proof as for Theorem 3.3. It is readily checked that problem (1.6) has a unique solution as a consequence of the coercivity of the bilinear form.

We consider the equation (1.6) with $v_{h}=u_{h}^{i+1}$ and we obtain :

$$
\frac{\alpha}{2}\left|u_{h}^{i+1}\right|_{1, \Omega}^{2}-\frac{\alpha}{2}\left|u_{h}^{i}\right|_{1, \Omega}^{2}+\frac{\alpha}{2}\left|u_{h}^{i+1}-u_{h}^{i}\right|_{1, \Omega}^{2}+\left|u_{h}^{i+1}\right|_{1, \Omega}^{2}+\lambda\left(\left|u_{h}^{i}\right|^{2 p} u_{h}^{i+1}, u_{h}^{i+1}\right)=\left(f, u_{h}^{i+1}\right) .
$$

We deduce the relation

$$
\frac{\alpha}{2}\left|u_{h}^{i+1}\right|_{1, \Omega}^{2}-\frac{\alpha}{2}\left|u_{h}^{i}\right|_{1, \Omega}^{2}+\frac{\alpha}{2}\left|u_{h}^{i+1}-u_{h}^{i}\right|_{1, \Omega}^{2}+\frac{1}{2}\left|u_{h}^{i+1}\right|_{1, \Omega}^{2}+\lambda\left(\left|u_{h}^{i}\right|^{2 p} u_{h}^{i+1}, u_{h}^{i+1}\right) \leq \frac{1}{2} \|\left. f\right|_{-1, \Omega} ^{2} .
$$

We prove the relation (3.6) recursively. Starting with (3.5), we suppose that we have

$$
\left|u_{h}^{i}\right|_{1, \Omega} \leq\|f\|_{-1, \Omega} .
$$

We are in one of the following two situations :

- We have $\left|u_{h}^{i+1}\right|_{1, \Omega} \leq\left|u_{h}^{i}\right|_{1, \Omega}$. We deduce the bound

$$
\left|u_{h}^{i+1}\right|_{1, \Omega} \leq \|\left. f\right|_{-1, \Omega} .
$$


- We have $\left|u_{h}^{i+1}\right|_{1, \Omega} \geq\left|u_{h}^{i}\right|_{1, \Omega}$. It follows from (3.7) that

$$
\left|u_{h}^{i+1}\right|_{1, \Omega}^{2} \leq\|f\|_{-1, \Omega}^{2} .
$$

We conclude the proof of the theorem.

Unfortunately the proof of the next result is much more technical.

Theorem 3.5. Assume that there exists $\beta>0$ such that, for every element $K \in \mathcal{T}_{h}$, we have

$$
h_{K} \geq \beta h,
$$

(which means that the family of triangulations is uniformly regular). Under the assumptions of Theorem 3.3 and for

$$
\alpha>C^{2} p^{2} \lambda^{2} h^{-4 p}
$$

where

$$
C=4 S_{4} S_{8} S_{8(2 p-1)}^{2 p-1} \frac{S_{I}^{2 p}}{\beta^{2 p}} S_{2}^{2 p}\|f\|_{-1, \Omega}^{2 p},
$$

the sequence of solutions $\left(u_{h}^{i}\right)$ of Problem (1.5) converges in $H_{0}^{1}(\Omega)$ to the solution $u_{h}$ of Problem (1.3).

Proof. We take the difference between the equations (1.5) and (1.3) with $v_{h}=u_{h}^{i+1}-u_{h}$ and we obtain the equation

$$
\begin{array}{r}
\frac{\alpha}{2}\left\|u_{h}^{i+1}-u_{h}\right\|_{0, \Omega}^{2}-\frac{\alpha}{2}\left\|u_{h}^{i}-u_{h}\right\|_{0, \Omega}^{2}+\frac{\alpha}{2}\left\|u_{h}^{i+1}-u_{h}^{i}\right\|_{0, \Omega}^{2}+\left|u_{h}^{i+1}-u_{h}\right|_{1, \Omega}^{2} \\
+\lambda\left(\left|u_{h}^{i}\right|^{2 p} u_{h}^{i+1}-\left|u_{h}\right|^{2 p} u_{h}, u_{h}^{i+1}-u_{h}\right)=0 .
\end{array}
$$

The last term in the previous equation, denoted by $T$, can be decomposed as

$$
T=\lambda\left(\left(\left|u_{h}^{i}\right|^{2 p}-\left|u_{h}^{i+1}\right|^{2 p}\right) u_{h}^{i+1}, u_{h}^{i+1}-u_{h}\right)+\lambda\left(\left|u_{h}^{i+1}\right|^{2 p} u_{h}^{i+1}-\left|u_{h}\right|^{2 p} u_{h}, u_{h}^{i+1}-u_{h}\right) .
$$

We denote by $T_{1}$ and $T_{2}$, respectively, the first and the second terms in the right-hand side of the last equation. Using Lemma 2.5, we have $T_{2} \geq 0$. Then we derive by using Lemma 2.4 (with $p$ replaced by $2 p$ )

$$
\begin{aligned}
& \frac{\alpha}{2}\left\|u_{h}^{i+1}-u_{h}\right\|_{0, \Omega}^{2}-\frac{\alpha}{2}\left\|u_{h}^{i}-u_{h}\right\|_{0, \Omega}^{2}+\frac{\alpha}{2}\left\|u_{h}^{i+1}-u_{h}^{i}\right\|_{0, \Omega}^{2}+\left|u_{h}^{i+1}-u_{h}\right|_{1, \Omega}^{2}+T_{2}=-T_{1} \\
& \quad \leq 2 p \lambda \int_{\Omega}\left(\left|u_{h}^{i+1}\right|^{2 p-1}+\left|u_{h}^{i}\right|^{2 p-1}\right)\left|u_{h}^{i+1}-u_{h}^{i}\right|\left|u_{h}^{i+1}\right|\left|u_{h}^{i+1}-u_{h}\right| d \mathbf{x} \\
& \quad \leq 2 p \lambda\left\|u_{h}^{i+1}-u_{h}^{i}\right\|_{0, \Omega}||\left|u_{h}^{i+1}\right|^{2 p-1}+\left|u_{h}^{i}\right|^{2 p-1}\left\|_{L^{8}(\Omega)}\right\| u_{h}^{i+1}\left\|_{L^{8}(\Omega)}\right\| u_{h}^{i+1}-u_{h} \|_{L^{4}(\Omega)} \\
& \quad \leq 2 p \lambda S_{4} S_{8} S_{8(2 p-1)}^{2 p-1}\left(\left|u_{h}^{i+1}\right|_{1, \Omega}^{2 p-1}+\left|u_{h}^{i}\right|_{1, \Omega}^{2 p-1}\right)\left|u_{h}^{i+1}\right|_{1, \Omega}\left\|u_{h}^{i+1}-u_{h}^{i}\right\|_{0, \Omega}\left|u_{h}^{i+1}-u_{h}\right|_{1, \Omega} \\
& \leq 2 p \lambda S_{4} S_{8} S_{8(2 p-1)}^{2 p-1} \frac{S_{I}^{2 p}}{\beta^{2 p}} h^{-2 p}|| u_{h}^{i+1}\left\|_{0, \Omega}^{2 p-1}+\right\| u_{h}^{i}\left\|_{0, \Omega}^{2 p-1}\right\| u_{h}^{i+1}\left\|_{0, \Omega}\right\| u_{h}^{i+1}-u_{h}^{i} \|_{0, \Omega}\left|u_{h}^{i+1}-u_{h}\right|_{1, \Omega} \\
& \leq 4 p \lambda S_{4} S_{8} S_{8(2 p-1)}^{2 p-1} \frac{S_{I}^{2 p}}{\beta^{2 p}} S_{2}^{2 p} h^{-2 p}\|f\|_{-1, \Omega}^{2 p}\left\|u_{h}^{i+1}-u_{h}^{i}\right\|_{0, \Omega}\left|u_{h}^{i+1}-u_{h}\right|_{1, \Omega}
\end{aligned}
$$

We denote by $C=4 S_{4} S_{8} S_{8(2 p-1)}^{2 p-1} \frac{S_{I}^{2 p}}{\beta^{2 p}} S_{2}^{2 p}\|f\|_{-1, \Omega}^{2 p}$, use the decomposition $a b \leq \frac{1}{2 \varepsilon} a^{2}+\frac{\varepsilon}{2} b^{2}$, take $\varepsilon=$ $\frac{1}{C p \lambda h^{-2 p}}$ and obtain the following bound

$\frac{\alpha}{2}\left\|u_{h}^{i+1}-u_{h}\right\|_{0, \Omega}^{2}-\frac{\alpha}{2}\left\|u_{h}^{i}-u_{h}\right\|_{0, \Omega}^{2}+\frac{\alpha}{2}\left\|u_{h}^{i+1}-u_{h}^{i}\right\|_{0, \Omega}^{2}+\frac{1}{2}\left|u_{h}^{i+1}-u_{h}\right|_{1, \Omega}^{2}+T_{2} \leq \frac{C^{2} p^{2} \lambda^{2}}{2} h^{-4 p}\left\|u_{h}^{i+1}-u_{h}^{i}\right\|_{0, \Omega}^{2}$.

We choice $\alpha>C^{2} p^{2} \lambda^{2} h^{-4 p}$, denote by $C_{1}=\frac{\alpha-C^{2} p^{2} \lambda^{2} h^{-4 p}}{2}$ and obtain

$$
\frac{\alpha}{2}\left\|u_{h}^{i+1}-u_{h}\right\|_{0, \Omega}^{2}-\frac{\alpha}{2}\left\|u_{h}^{i}-u_{h}\right\|_{0, \Omega}^{2}+C_{1}\left\|u_{h}^{i+1}-u_{h}^{i}\right\|_{0, \Omega}^{2}+\frac{1}{2}\left|u_{h}^{i+1}-u_{h}\right|_{1, \Omega}^{2}+T_{2} \leq 0 .
$$

We deduce that, for all $i \geq 1$, we have (if $\left\|u_{h}^{i}-u_{h}\right\|_{0, \Omega} \neq 0$ )

$$
\left\|u_{h}^{i+1}-u_{h}\right\|_{0, \Omega}<\left\|u_{h}^{i}-u_{h}\right\|_{0, \Omega}
$$


and we deduce the convergence of the sequence $\left(u_{h}^{i+1}-u_{h}\right)$ in $L^{2}(\Omega)$. By taking the limit of (3.9) we deduce that $\left|u_{h}^{i+1}-u_{h}\right|_{1, \Omega}$ converges to 0 and $u_{h}^{i+1}$ converges to $u_{h}$ in $H_{0}^{1}(\Omega)$.

Theorem 3.6. Under the assumptions of Theorem 3.4 and for

$$
\alpha>\left(4 S_{2} S_{4} S_{8} S_{8(2 p-1)}^{2 p-1}\|f\|_{-1, \Omega}^{2 p}\right)^{2} p^{2} \lambda^{2},
$$

the sequence of solutions $\left(u_{h}^{i}\right)$ of Problem (1.6) converges in $H_{0}^{1}(\Omega)$ to the solution $u_{h}$ of Problem (1.3).

Proof. We take the difference between the equations (1.6) and (1.3) with $v_{h}=u_{h}^{i+1}-u_{h}$ and we obtain the equation

$$
\begin{aligned}
\frac{\alpha}{2}\left|u_{h}^{i+1}-u_{h}\right|_{1, \Omega}^{2}-\frac{\alpha}{2}\left|u_{h}^{i}-u_{h}\right|_{1, \Omega}^{2} & +\frac{\alpha}{2}\left|u_{h}^{i+1}-u_{h}^{i}\right|_{1, \Omega}^{2}+\left|u_{h}^{i+1}-u_{h}\right|_{1, \Omega}^{2} \\
& +\lambda\left(\left|u_{h}^{i}\right|^{2 p} u_{h}^{i+1}-\left|u_{h}\right|^{2 p} u_{h}, u_{h}^{i+1}-u_{h}\right)=0 .
\end{aligned}
$$

The last term in the previous equation, denoted by $T$, can be decomposed as

$$
T=\lambda\left(\left(\left|u_{h}^{i}\right|^{2 p}-\left|u_{h}^{i+1}\right|^{2 p}\right) u_{h}^{i+1}, u_{h}^{i+1}-u_{h}\right)+\lambda\left(\left|u_{h}^{i+1}\right|^{2 p} u_{h}^{i+1}-\left|u_{h}\right|^{2 p} u_{h}, u_{h}^{i+1}-u_{h}\right) .
$$

We denote by $T_{1}$ and $T_{2}$ respectively the first and the second terms in the right-hand side of the last equation. Using Lemma 2.5, we have $T_{2} \geq 0$. Then we have by using Lemma 2.4

$$
\begin{aligned}
\frac{\alpha}{2} \mid u_{h}^{i+1} & -\left.u_{h}\right|_{1, \Omega} ^{2}-\frac{\alpha}{2}\left|u_{h}^{i}-u_{h}\right|_{1, \Omega}^{2}+\frac{\alpha}{2}\left|u_{h}^{i+1}-u_{h}^{i}\right|_{1, \Omega}^{2}+\left|u_{h}^{i+1}-u_{h}\right|_{1, \Omega}^{2}+T_{2}=-T_{1} \\
& \leq 2 p \lambda\left(\left(\left|u_{h}^{i+1}\right|^{2 p-1}+\left|u_{h}^{i}\right|^{2 p-1}\right),\left|u_{h}^{i+1}-u_{h}^{i}\right|\left|u_{h}^{i+1}\right|\left|u_{h}^{i+1}-u_{h}\right|\right) \\
& \leq\left. 2 p \lambda\left\|u_{h}^{i+1}-\left.u_{h}^{i}\right|_{0, \Omega}\right\|\left(\left|u_{h}^{i+1}\right|{ }^{2 p-1}+\left|u_{h}^{i}\right|^{2 p-1}\right)\right|_{L^{8}(\Omega)}\left\|\left.u_{h}^{i+1}\right|_{L^{8}(\Omega)}\right\| u_{h}^{i+1}-\left.u_{h}\right|_{L^{4}(\Omega)} \\
& \leq 4 p \lambda S_{2} S_{4} S_{8} S_{8(2 p-1)}^{2 p-1}\|f\|_{-1, \Omega}^{2 p}\left|u_{h}^{i+1}-u_{h}^{i}\right|_{1, \Omega}\left|u_{h}^{i+1}-u_{h}\right|_{1, \Omega} .
\end{aligned}
$$

We denote by $C=4 S_{2} S_{4} S_{8} S_{8(2 p-1)}^{2 p-1}\|f\|_{-1, \Omega}^{2 p}$, use the decomposition $a b \leq \frac{1}{2 \varepsilon} a^{2}+\frac{\varepsilon}{2} b^{2}$, take $\varepsilon=\frac{1}{C p \lambda}$ and obtain the following bound

$$
\frac{\alpha}{2}\left|u_{h}^{i+1}-u_{h}\right|_{1, \Omega}^{2}-\frac{\alpha}{2}\left|u_{h}^{i}-u_{h}\right|_{1, \Omega}^{2}+\frac{\alpha}{2}\left|u_{h}^{i+1}-u_{h}^{i}\right|_{1, \Omega}^{2}+\frac{1}{2}\left|u_{h}^{i+1}-u_{h}\right|_{1, \Omega}^{2}+T_{2} \leq \frac{C^{2} p^{2} \lambda^{2}}{2}\left|u_{h}^{i+1}-u_{h}^{i}\right|_{1, \Omega}^{2} .
$$

We choose $\alpha>C^{2} p^{2} \lambda^{2}$, denote by $C_{1}=\frac{\alpha-C^{2} p^{2} \lambda^{2}}{2}$ and obtain

$$
\frac{\alpha}{2}\left|u_{h}^{i+1}-u_{h}\right|_{1, \Omega}^{2}-\frac{\alpha}{2}\left|u_{h}^{i}-u_{h}\right|_{1, \Omega}^{2}+C_{1}\left|u_{h}^{i+1}-u_{h}^{i}\right|_{1, \Omega}^{2}+\frac{1}{2}\left|u_{h}^{i+1}-u_{h}\right|_{1, \Omega}^{2}+T_{2} \leq 0 .
$$

We derive that, for all $i \geq 1$, we have

$$
\left|u_{h}^{i+1}-u_{h}\right|_{1, \Omega}<\left|u_{h}^{i}-u_{h}\right|_{1, \Omega},
$$

we obtain the convergence of the sequence $\left(u_{h}^{i+1}-u_{h}\right)$ in $H^{1}(\Omega)$ and, by taking the limit of (3.11) we deduce that $u_{h}^{i+1}$ converges to $u_{h}$ in $H_{0}^{1}(\Omega)$.

Remark 3.7. The conditions (3.2) and (3.5) suppose that the initial values of the algorithms are small related to the data $f$. We can always take $u_{h}^{0}=0$.

Remark 3.8. The previous two theorems bring to light a first difference between the two schemes (1.5) and (1.6): in opposite to (1.5), the convergence of (1.6) is proved when $\alpha$ is larger than a constant independent of $h$ (and does not require the uniform regularity of the family of triangulations).

\section{A POSTERIORI ERROR ANALYSIS}

We start this section by introducing some additional notation which is needed for constructing and analyzing the error indicators in the sequel.

For any triangle $K \in \mathcal{T}_{h}$ we denote by $\mathcal{E}(K)$ and $\mathcal{N}(K)$ the set of its edges and vertices, respectively, and we set

$$
\mathcal{E}_{h}=\bigcup_{K \in \mathcal{T}_{h}} \mathcal{E}(K) \quad \text { and } \quad \mathcal{N}_{h}=\bigcup_{K \in \mathcal{T}_{h}} \mathcal{N}(K) .
$$


With any edge $e \in \mathcal{E}_{h}$ we associate a unit vector $n$ such that $n$ is orthogonal to $e$. We split $\mathcal{E}_{h}$ and $\mathcal{N}_{h}$ in the form

$$
\mathcal{E}_{h}=\mathcal{E}_{h, \Omega} \cup \mathcal{E}_{h, \partial \Omega} \quad \text { and } \quad \mathcal{N}_{h}=\mathcal{N}_{h, \Omega} \cup \mathcal{N}_{h, \partial \Omega}
$$

where $\mathcal{E}_{h, \partial \Omega}$ is the set of edges in $\mathcal{E}_{h}$ that lie on $\partial \Omega$ and $\mathcal{E}_{h, \Omega}=\mathcal{E}_{h} \backslash \mathcal{E}_{h, \partial \Omega}$. The same goes for $\mathcal{N}_{h, \partial \Omega}$.

Furthermore, for $K \in \mathcal{T}_{h}$ and $e \in \mathcal{E}_{h}$, let $h_{K}$ and $h_{e}$ be their diameter and length, respectively. An important tool in the construction of an upper bound for the total error is Clément's interpolation operator $\mathcal{R}_{h}$ with values in $V_{h}$. The operator $\mathcal{R}_{h}$ satisfies, for all $v \in H_{0}^{1}(\Omega)$, the following local approximation properties (see R. Verfürth, [7], Chapter 1):

$$
\begin{aligned}
& \left\|v-R_{h} v\right\|_{L^{2}(K)} \leq C h_{K}|v|_{1, \Delta_{K}}, \\
& \left\|v-R_{h} v\right\|_{L^{2}(e)} \leq C h_{e}^{1 / 2}|v|_{1, \Delta_{e}},
\end{aligned}
$$

where $\Delta_{K}$ and $\Delta_{e}$ are the following sets:

$$
\Delta_{K}=\bigcup\left\{K^{\prime} \in \mathcal{T}_{h} ; K^{\prime} \cap K \neq \emptyset\right\} \quad \text { and } \quad \Delta_{e}=\bigcup\left\{K^{\prime} \in \mathcal{T}_{h} ; \quad K^{\prime} \cap e \neq \emptyset\right\} .
$$

We now recall the following properties (see R. Verfürth, [7], Chapter 1):

Proposition 4.1. Let $r$ be a positive integer. For all $v \in P_{r}(K)$, the following properties hold

$$
\begin{gathered}
C\|v\|_{L^{2}(K)} \leq\left\|v \psi_{K}^{1 / 2}\right\|_{L^{2}(K)} \leq\|v\|_{L^{2}(K)}, \\
|v|_{1, K} \leq C h_{K}^{-1}\|v\|_{L^{2}(K)} .
\end{gathered}
$$

where $\psi_{K}$ is the triangle-bubble function (equal to the product of the barycentric coordinates associated with the vertices of $K$ ).

We also introduce a lifting operator: For each $K \in \mathcal{T}_{h}$ and any edge $e$ of $K, L_{e, K}$ maps polynomials of fixed degree on $e$ vanishing on $\partial e$ into polynomials on $K$ vanishing on $\partial K \backslash e$ and is constructed by affine transformation from a fixed lifting operator on the reference triangle.

Proposition 4.2. Let $r$ be a positive integer. For all $v \in P_{r}(e)$, we have the following property

$$
C\|v\|_{L^{2}(e)} \leq\left\|v \psi_{e}^{1 / 2}\right\|_{L^{2}(e)} \leq\|v\|_{L^{2}(e)},
$$

where $\psi_{e}$ is the bubble function on the edge e, and for all $v \in P_{r}(e)$ vanishing on $\partial e$, we have

$$
\left\|L_{e, \kappa} v\right\|_{L^{2}(\kappa)}+h_{e}\left|L_{e, \kappa} v\right|_{1, \kappa} \leq C h_{e}^{1 / 2}\|v\|_{L^{2}(e)},
$$

where $\kappa$ is a triangle of edge $e$.

Finally, we denote by $\left[v_{h}\right]$ the jump of $v_{h}$ across the common edge $e$ of two adjacent elements $K, K^{\prime} \in \mathcal{T}_{h}$. We have now provided all prerequisites to establish an upper bound and lower bound for the total error. Let $u_{h}^{i+1}$ and $u$ be the solution of the iterative problem (1.5) or (1.6) and the continuous problem, respectively. They satisfy the identity

$$
\int_{\Omega} \nabla\left(u_{h}^{i+1}-u\right) \nabla v d \mathbf{x}=\int_{\Omega} \nabla u_{h}^{i+1} \nabla v d \mathbf{x}+\lambda \int_{\Omega}|u|^{2 p} u v d \mathbf{x}-\int_{\Omega} f v d \mathbf{x} .
$$

We now start the a posteriori analysis of our algorithms.

\subsection{Algorithm (1.5).}

In order to prove an upper bound of the error, we introduce an approximation $f_{h}$ of the data $f$ which is constant on each element $K$ of $\mathcal{T}_{h}$. We first write the residual equation

$$
\begin{aligned}
& \int_{\Omega} \nabla u \nabla v d \mathbf{x}+\lambda \int_{\Omega}|u|^{2 p} u v d \mathbf{x}-\int_{\Omega} \nabla u_{h}^{i+1} \nabla v d \mathbf{x}-\lambda \int_{\Omega}\left|u_{h}^{i}\right|^{2 p} u_{h}^{i+1} v d \mathbf{x} \\
& =\int_{\Omega}\left(f-f_{h}\right)\left(v-v_{h}\right) d \mathbf{x}+\sum_{K \in \mathcal{T}_{h}}\left\{\int_{K}\left(f_{h}+\Delta u_{h}^{i+1}-\lambda\left|u_{h}^{i}\right|^{2 p} u_{h}^{i+1}-\alpha\left(u_{h}^{i+1}-u_{h}^{i}\right)\right)\left(v-v_{h}\right) d \mathbf{x}\right. \\
& \left.\quad-\frac{1}{2} \sum_{e \in \mathcal{E}(K)} \int_{e}\left[\frac{\partial u_{h}^{i+1}}{\partial n}\right]\left(v-v_{h}\right) d \tau\right\}+\alpha \sum_{K \in \mathcal{T}_{h}} \int_{K}\left(u_{h}^{i+1}-u_{h}^{i}\right) v d \mathbf{x} .
\end{aligned}
$$


By adding and subtracting $\lambda \int_{\Omega}\left|u_{h}^{i+1}\right|^{2 p} u_{h}^{i+1} v d \mathbf{x}$, we obtain

$$
\begin{aligned}
& \int_{\Omega} \nabla u \nabla v d \mathbf{x}+\lambda \int_{\Omega}|u|^{2 p} u v d \mathbf{x}-\int_{\Omega} \nabla u_{h}^{i+1} \nabla v d \mathbf{x}-\lambda \int_{\Omega}\left|u_{h}^{i+1}\right|^{2 p} u_{h}^{i+1} v d \mathbf{x} \\
& =\int_{\Omega}\left(f-f_{h}\right)\left(v-v_{h}\right) d \mathbf{x}+\sum_{K \in \mathcal{T}_{h}}\left\{\int_{K}\left(f_{h}+\Delta u_{h}^{i+1}-\lambda\left|u_{h}^{i}\right|^{2 p} u_{h}^{i+1}-\alpha\left(u_{h}^{i+1}-u_{h}^{i}\right)\right)\left(v-v_{h}\right) d \mathbf{x}\right. \\
& \left.-\frac{1}{2} \sum_{e \in \mathcal{E}(K)} \int_{e}\left[\frac{\partial u_{h}^{i+1}}{\partial n}\right]\left(v-v_{h}\right) d \tau\right\}+\lambda \int_{\Omega}\left(\left|u_{h}^{i}\right|^{2 p}-\left|u_{h}^{i+1}\right|^{2 p}\right) u_{h}^{i+1} v d \mathbf{x}+\alpha \sum_{K \in \mathcal{T}_{h}} \int_{K}\left(u_{h}^{i+1}-u_{h}^{i}\right) v d \mathbf{x} .
\end{aligned}
$$

We now define the local linearization indicator $\eta_{K, i}^{(L)}$ and the local discretization indicator $\eta_{K, i}^{(D)}$ at each iteration $i$ by:

$$
\begin{aligned}
\eta_{K, i}^{(L)} & =\left\|u_{h}^{i+1}-u_{h}^{i}\right\|_{1, K}, \\
\left(\eta_{K, i}^{(D)}\right)^{2} & =h_{K}^{2}\left\|f_{h}+\Delta u_{h}^{i+1}-\lambda\left|u_{h}^{i}\right|^{2 p} u_{h}^{i+1}-\alpha\left(u_{h}^{i+1}-u_{h}^{i}\right)\right\|_{L^{2}(K)}^{2}+\sum_{e \in \mathcal{E}(K)} h_{e}\left\|\left[\frac{\partial u_{h}^{i+1}}{\partial n}\right]\right\|_{L^{2}(e)}^{2} .
\end{aligned}
$$

We are in a position to state the first result of this section:

Theorem 4.3. Upper bound. Let $u_{h}^{i+1}$ and $u$ be the solution of the iterative problem (1.5) and the exact problem (2.2) respectively. We have the following a posteriori error estimate

$$
\left|u_{h}^{i+1}-u\right|_{1, \Omega} \leq C\left(\left(\sum_{K \in \mathcal{T}_{h}}\left(\left(\eta_{K, i}^{(D)}\right)^{2}+h_{K}^{2}\left\|f-f_{h}\right\|_{L^{2}(K)}^{2}\right)\right)^{1 / 2}+\left(\sum_{K \in \mathcal{T}_{h}}\left(\eta_{K, i}^{(L)}\right)^{2}\right)^{1 / 2}\right) .
$$

Proof. We consider equation (4.7) with $v=u-u_{h}^{i+1}$ and we obtain

$$
\begin{aligned}
& \int_{\Omega} \nabla\left(u-u_{h}^{i+1}\right)^{2} d \mathbf{x}+\lambda \int_{\Omega}\left(|u|^{2 p} u-\left|u_{h}^{i+1}\right|^{2 p} u_{h}^{i+1}\right)\left(u-u_{h}^{i+1}\right) d \mathbf{x} \\
& =\sum_{K \in \mathcal{T}_{h}} \int_{K}\left(f-f_{h}\right)\left(v-v_{h}\right) d \mathbf{x}+\sum_{K \in \mathcal{T}_{h}}\left\{\int_{K}\left(f_{h}+\Delta u_{h}^{i+1}-\lambda\left|u_{h}^{i}\right|^{2 p} u_{h}^{i+1}-\alpha\left(u_{h}^{i+1}-u_{h}^{i}\right)\right)\left(v-v_{h}\right) d \mathbf{x}\right. \\
& \left.-\frac{1}{2} \sum_{e \in \mathcal{E}(K)} \int_{e}\left[\frac{\partial u_{h}^{i+1}}{\partial n}\right]\left(v-v_{h}\right) d \tau\right\}+\lambda \int_{\Omega}\left(\left|u_{h}^{i}\right|^{2 p}-\left|u_{h}^{i+1}\right|^{2 p}\right) u_{h}^{i+1} v d \mathbf{x}+\alpha \sum_{K \in \mathcal{T}_{h}} \int_{K}\left(u_{h}^{i+1}-u_{h}^{i}\right) v d \mathbf{x} .
\end{aligned}
$$

Then we have by using Lemmas 2.4 and 2.5

$$
\begin{aligned}
& \left|u-u_{h}^{i+1}\right|_{1, \Omega}^{2} \leq \sum_{K \in \mathcal{T}_{h}}\left\|f-f_{h}\right\|_{L^{2}(K)}|| v-v_{h} \|_{L^{2}(K)} \\
& \quad+\sum_{K \in \mathcal{T}_{h}}\left(\left\|f_{h}+\Delta u_{h}^{i+1}-\lambda\left|u_{h}^{i}\right|^{2 p} u_{h}^{i+1}-\alpha\left(u_{h}^{i+1}-u_{h}^{i}\right)\right\|_{L^{2}(K)}|| v-v_{h} \|_{L^{2}(K)}\right. \\
& \left.\quad+\frac{1}{2} \sum_{e \in \mathcal{E}(K)}\left\|\left[\frac{\partial u_{h}^{i+1}}{\partial n}\right]\right\|_{L^{2}(e)}\left\|v-v_{h}\right\|_{L^{2}(e}\right)+\lambda \int_{\Omega} 2 p\left|u_{h}^{i}-u_{h}^{i+1}\right|\left(\left|u_{h}^{i}\right|^{2 p-1}+\left|u_{h}^{i+1}\right|^{2 p-1}\right)\left|u_{h}^{i+1}\right||v| d \mathbf{x} \\
& \quad+\alpha \sum_{K \in \mathcal{T}_{h}}\left\|u_{h}^{i+1}-u_{h}^{i}\right\|_{L^{2}(K)}\|v\|_{L^{2}(K)}
\end{aligned}
$$

We choose $v_{h}=R_{h} v$, the image of $v$ by the Clément operator and we obtain

$$
\begin{aligned}
& \left|u-u_{h}^{i+1}\right|_{1, \Omega}^{2} \leq C \sum_{K \in \mathcal{T}_{h}} h_{K}|| f-\left.f_{h}\right|_{L^{2}(K)}|v|_{1, \Delta_{K}} \\
& +\sum_{K \in \mathcal{T}_{h}}\left(C h_{K}|| f_{h}+\Delta u_{h}^{i+1}-\lambda\left|u_{h}^{i}\right|^{2 p} u_{h}^{i+1}-\alpha\left(u_{h}^{i+1}-u_{h}^{i}\right)||_{L^{2}(K)}|v|_{1, \Delta_{K}}\right. \\
& \left.\quad+\frac{C}{2} \sum_{e \in \mathcal{E}(K)} h_{e}^{\frac{1}{2}}||\left[\frac{\partial u_{h}^{i+1}}{\partial n}\right]||_{L^{2}(e)}|v|_{1, \Delta_{e}}\right) \\
& \quad+\lambda \int_{\Omega} 2 p\left|u_{h}^{i}-u_{h}^{i+1}\right|\left(\left|u_{h}^{i}\right|^{2 p-1}+\left|u_{h}^{i+1}\right|^{2 p-1}\right)\left|u_{h}^{i+1}\right||v| d \mathbf{x}+\alpha \sum_{K \in \mathcal{T}_{h}}\left\|u_{h}^{i+1}-u_{h}^{i}\right\|_{L^{2}(K)}\|v\|_{L^{2}(K)}
\end{aligned}
$$


We begin by bounding the second term of the right-hand side of the last inequality and we obtain by using Theorem 3.3

$$
\begin{aligned}
& \lambda \int_{\Omega} 2 p\left|u_{h}^{i}-u_{h}^{i+1}\right|\left(\left|u_{h}^{i}\right|^{2 p-1}+\left|u_{h}^{i+1}\right|^{2 p-1}\right)\left|u_{h}^{i+1}\right||v| d \mathbf{x} \\
& \leq 2 \lambda p\left\|\left.|| u_{h}^{i}\right|^{2 p-1}+\left|u_{h}^{i+1}\right|^{2 p-1}||_{L^{8}(\Omega)}|| u_{h}^{i}-u_{h}^{i+1}||_{L^{8}(\Omega)}|| u_{h}^{i+1}\right\|_{L^{4}(\Omega)}|| v \|_{L^{2}(\Omega)} \\
& \leq 2 \lambda p S_{2} S_{4} S_{8}\left(\|\left.\left|u_{h}^{i}\right|^{2 p-1}\right|_{L^{8}(\Omega)}+||\left|u_{h}^{i+1}\right|{ }^{2 p-1}||_{L^{8}(\Omega)}\right)\left|u_{h}^{i}-u_{h}^{i+1}\right|_{1, \Omega}\left|u_{h}^{i+1}\right|_{1, \Omega}|v|_{1, \Omega} \\
& \leq 2 \lambda p S_{2} S_{4} S_{8} S_{8(2 p-1)}^{2 p-1}\left(\left|u_{h}^{i}\right|_{1, \Omega}^{2 p-1}+\left|u_{h}^{i+1}\right|_{1, \Omega}^{2 p-1}\right)\left|u_{h}^{i}-u_{h}^{i+1}\right|_{1, \Omega}\left|u_{h}^{i+1}\right|_{1, \Omega}|v|_{1, \Omega} \\
& \leq\left. 4 \lambda p\left(1+\alpha S_{2}^{2}\right)^{p} S_{2} S_{4} S_{8} S_{8(2 p-1)}^{2 p-1}|| f\right|_{-1, \Omega} ^{2 p}\left|u_{h}^{i}-u_{h}^{i+1}\right|_{1, \Omega}|v|_{1, \Omega}
\end{aligned}
$$

Let $S=4 \lambda p\left(1+\alpha S_{2}^{2}\right)^{p} S_{2} S_{4} S_{8} S_{8(2 p-1)}^{2 p-1}\|f\|_{-1, \Omega}^{2 p}$, then we have

$$
\begin{aligned}
& \left|u-u_{h}^{i+1}\right|_{1, \Omega}^{2} \leq C \sum_{K \in \mathcal{T}_{h}} h_{K}|| f-f_{h} \|_{L^{2}(K)}|v|_{1, \Delta_{K}} \\
& +\sum_{K \in \mathcal{T}_{h}}\left(C h_{K}|| f_{h}+\Delta u_{h}^{i+1}-\lambda\left|u_{h}^{i}\right|^{2 p} u_{h}^{i+1}-\alpha\left(u_{h}^{i+1}-u_{h}^{i}\right)||_{L^{2}(K)}|v|_{1, \Delta_{K}}\right. \\
& \left.+\frac{C}{2} \sum_{e \in \mathcal{E}_{(K)}} h_{e}^{\frac{1}{2}}||\left[\frac{\partial u_{h}^{i+1}}{\partial n}\right]||_{L^{2}(e)}|v|_{1, \Delta_{e}}\right) \\
& +S\left|u_{h}^{i}-u_{h}^{i+1}\right|_{1, \Omega}|v|_{1, \Omega}+\alpha \sum_{K \in \mathcal{T}_{h}}\left\|u_{h}^{i+1}-u_{h}^{i}\right\|_{L^{2}(K)}|| v \|_{L^{2}(K)} .
\end{aligned}
$$

By using the formula $a b \leq \frac{1}{2 \varepsilon} a^{2}+\frac{\varepsilon}{2} b^{2}$, we obtain

$$
\begin{aligned}
\left|u-u_{h}^{i+1}\right|_{1, \Omega}^{2} \leq & \frac{C \varepsilon_{1}}{2} \sum_{K \in \mathcal{T}_{h}} h_{K}^{2}\left\|f-f_{h}\right\|_{L^{2}(K)}^{2}+\frac{C_{1}}{2 \varepsilon_{1}} \sum_{K \in \mathcal{T}_{h}}|v|_{1, K}^{2} \\
& +\frac{C \varepsilon_{2}}{2} \sum_{K \in \mathcal{T}_{h}} h_{K}^{2}\left\|f_{h}+\Delta u_{h}^{i+1}-\lambda\left|u_{h}^{i}\right|^{2 p} u_{h}^{i+1}-\alpha\left(u_{h}^{i+1}-u_{h}^{i}\right)\right\|_{L^{2}(K)}^{2}+\frac{C_{2}}{2 \varepsilon_{2}} \sum_{K \in \mathcal{T}_{h}}|v|_{1, K}^{2} \\
& +\left.\frac{C \varepsilon_{3}}{4} \sum_{K \in \mathcal{T}_{h}} \sum_{E \in \mathcal{E}_{(K)}} h_{E}\left|\left\|\left[\frac{\partial u_{h}^{i+1}}{\partial n}\right]\right\|_{L^{2}(E)}^{2}+\frac{C_{3}}{4 \varepsilon_{3}} \sum_{K \in \mathcal{T}_{h}}\right| v\right|_{1, K} ^{2} \\
& +\frac{S \varepsilon_{4}}{2} \sum_{K \in \mathcal{T}_{h}}\left\|u_{h}^{i}-u_{h}^{i+1}\right\|_{1, K}^{2}+\frac{S}{2 \varepsilon_{4}} \sum_{K \in \mathcal{T}_{h}}|v|_{1, K}^{2} \\
& +\frac{\alpha \varepsilon_{5}}{2} \sum_{K \in \mathcal{T}_{h}}\left\|u_{h}^{i+1}-u_{h}^{i}\right\|_{1, K}^{2}+\frac{\alpha}{2 \varepsilon_{5}}\|v\|_{L^{2}(\Omega)}^{2}
\end{aligned}
$$

We choose $\varepsilon_{1}=8 C_{1}, \varepsilon_{2}=8 C_{2}, \varepsilon_{3}=4 C_{3}, \varepsilon_{4}=8 S$ et $\varepsilon_{5}=8 \alpha S_{2}^{2}$ to obtain

$$
\begin{aligned}
\left|u-u_{h}^{i+1}\right|_{1, \Omega}^{2} \leq & C\left(\sum_{K \in \mathcal{T}_{h}} h_{K}^{2}\left\|f-f_{h}\right\|_{L^{2}(K)}^{2}+\sum_{K \in \mathcal{T}_{h}} \sum_{e \in \mathcal{E}_{(K)}} h_{e}\left\|\left[\frac{\partial u_{h}^{i+1}}{\partial n}\right]\right\|_{L^{2}(e)}^{2}\right. \\
& +\sum_{K \in \mathcal{T}_{h}} h_{K}^{2}\left\|f_{h}+\Delta u_{h}^{i+1}-\lambda\left|u_{h}^{i}\right|^{2 p} u_{h}^{i+1}-\alpha\left(u_{h}^{i+1}-u_{h}^{i}\right)\right\|_{L^{2}(K)}^{2} \\
& \left.+\sum_{K \in \mathcal{T}_{h}}\left\|u_{h}^{i}-u_{h}^{i+1}\right\|_{1, K}^{2}+\sum_{K \in \mathcal{T}_{h}}\left\|u_{h}^{i+1}-u_{h}^{i}\right\|_{1, K}^{2}\right)+\frac{5}{16}|v|_{1, \Omega}^{2}
\end{aligned}
$$

and then

$$
\left|u_{h}^{i+1}-u\right|_{1, \Omega} \leq C\left(\left(\sum_{K \in \mathcal{T}_{h}}\left(\left(\eta_{K, i}^{(D)}\right)^{2}+h_{K}^{2}\left\|f-f_{h}\right\|_{L^{2}(K)}^{2}\right)\right)^{\frac{1}{2}}+\left(\sum_{K \in \mathcal{T}_{h}}\left(\eta_{K, i}^{(L)}\right)^{2}\right)^{\frac{1}{2}}\right) .
$$

We conclude the proof of the theorem. 
We address now the efficiency of the previous indicators.

Theorem 4.4. Lower bound. For each $K \in \mathcal{T}_{h}$, there holds

$$
\begin{aligned}
& \eta_{K, i}^{(L)} \leq\left\|u_{h}^{i}-u\right\|_{1, K}+\left\|u_{h}^{i+1}-u\right\|_{1, K}, \\
& \eta_{K, i}^{(D)} \leq C \sum_{\kappa \subset \omega_{K}}\left(\left\|u-u_{h}^{i+1}\right\|_{1, \kappa}+\eta_{\kappa, i}^{(L)}+h_{\kappa}\left\|f-f_{h}\right\|_{L^{2}(\kappa)}\right),
\end{aligned}
$$

where $\omega_{K}$ is the union of the triangles sharing at least one edge with $K$.

Proof. The estimation of the linearization indicator follows easily from the triangle inequality by introducing $u$ in $\eta_{K, i}^{(L)}$. We now estimate the discretization indicator $\eta_{K, i}^{(D)}$. We proceed in two steps:

(i) We start by adding and subtracting $\lambda \int_{\Omega}\left|u_{h}^{i+1}\right|^{2 p} u_{h}^{i+1} v d \mathbf{x}$ in (4.6). Taking $v_{h}=0$, we derive

$$
\begin{aligned}
\sum_{K \in \mathcal{T}_{h}} \int_{K}\left(f_{h}+\Delta u_{h}^{i+1}-\lambda\left|u_{h}^{i}\right|^{2 p} u_{h}^{i+1}-\alpha\left(u_{h}^{i+1}-u_{h}^{i}\right)\right) v d \mathbf{x} \\
=\int_{\Omega} \nabla\left(u-u_{h}^{i+1}\right) \nabla v d \mathbf{x}-\sum_{K \in \mathcal{T}_{h}} \int_{K}\left(f-f_{h}\right) v d \mathbf{x} \\
+\frac{1}{2} \sum_{K \in \mathcal{T}_{h}} \sum_{e \in \mathcal{E}(K)} \int_{e}\left[\frac{\partial u_{h}^{i+1}}{\partial n}\right] v d \tau+\lambda \int_{\Omega}\left(|u|^{2 p} u-\left|u_{h}^{i+1}\right|^{2 p} u_{h}^{i+1}\right) v d \mathbf{x} \\
+\lambda \int_{\Omega} u_{h}^{i+1}\left(\left|u_{h}^{i+1}\right|^{2 p}-\left|u_{h}^{i}\right|^{2 p}\right) v d \mathbf{x}-\alpha \sum_{K \in \mathcal{T}_{h}} \int_{K}\left(u_{h}^{i+1}-u_{h}^{i}\right) v d \mathbf{x} .
\end{aligned}
$$

We choose $v=v_{K}$ such that

$$
v_{K}=\left\{\begin{array}{cl}
\left(f_{h}+\Delta u_{h}^{i+1}-\lambda\left|u_{h}^{i}\right|^{2 p} u_{h}^{i+1}-\alpha\left(u_{h}^{i+1}-u_{h}^{i}\right)\right) \psi_{K} & \text { in } K \\
0 & \text { in } \Omega \backslash K
\end{array}\right.
$$

where $\psi_{K}$ is the triangle-bubble function.

Using Cauchy-Schwarz inequality, (2.1), (4.1) and (4.2) we obtain

$$
\begin{aligned}
& \left\|f_{h}+\Delta u_{h}^{i+1}-\lambda\left|u_{h}^{i}\right|^{2 p} u_{h}^{i+1}-\alpha\left(u_{h}^{i+1}-u_{h}^{i}\right)\right\|_{L^{2}(K)}^{2} \\
& \leq\left(1+\lambda C\|f\|_{-1, \Omega}^{2 p}\right)\left\|u-u_{h}^{i+1}\right\|_{1, K}\left|v_{K}\right|_{1, K}+\left\|f-f_{h}\right\|_{L^{2}(K)}\left\|v_{K}\right\|_{L^{2}(K)} \\
& \quad+\lambda C\left\|u_{h}^{i}-u_{h}^{i+1}\right\|_{1, K}\left|v_{K}\right|_{1, K}+\alpha\left\|u_{h}^{i+1}-u_{h}^{i}\right\|_{L^{2}(K)}\left\|v_{K}\right\|_{L^{2}(K)} .
\end{aligned}
$$

Therefore, we derive the following estimate of the first term of the local discretization estimator $\eta_{K, i}^{(D)}$

$$
\begin{aligned}
h_{K} \| f_{h}+\Delta u_{h}^{i+1} & -\lambda\left|u_{h}^{i}\right|^{2 p} u_{h}^{i+1}-\alpha\left(u_{h}^{i+1}-u_{h}^{i}\right) \|_{L^{2}(K)} \\
& \leq C\left(\left\|u-u_{h}^{i+1}\right\|_{1, K}+h_{K}\left\|f-f_{h}\right\|_{L^{2}(K)}\right)+C^{\prime} \eta_{K, i}^{(L)}
\end{aligned}
$$

(ii) Now we estimate the second term of $\eta_{K, i}^{(D)}$. Similarly, using (4.9) we infer

$$
\begin{aligned}
& \frac{1}{2} \sum_{K \in \mathcal{T}_{h}} \sum_{e \in \mathcal{E}(K)} \int_{e}\left[\frac{\partial u_{h}^{i+1}}{\partial n}\right] v d \tau=\int_{\Omega} \nabla\left(u_{h}^{i+1}-u\right) \nabla v d \mathbf{x} \\
& \quad+\sum_{K \in \mathcal{T}_{h}} \int_{K}\left(f_{h}+\Delta u_{h}^{i+1}-\lambda\left|u_{h}^{i}\right|^{2 p} u_{h}^{i+1}-\alpha\left(u_{h}^{i+1}-u_{h}^{i}\right)\right) v d \mathbf{x}+\int_{\Omega}\left(f-f_{h}\right) v d \mathbf{x} \\
& \quad-\lambda \int_{\Omega}\left(\left|u_{h}^{i+1}\right|^{2 p} u_{h}^{i+1}-\left|u_{h}^{i}\right|^{2 p} u_{h}^{i+1}\right) v d \mathbf{x}-\lambda \int_{\Omega}\left(|u|^{2 p} u-\left|u_{h}^{i+1}\right|^{2 p} u_{h}^{i+1}\right) v d \mathbf{x}+\alpha \sum_{K \in \mathcal{T}_{h}} \int_{K}\left(u_{h}^{i+1}-u_{h}^{i}\right) v d \mathbf{x} .
\end{aligned}
$$


We choose $v=v_{e}$ such that

$$
v_{e}=\left\{\begin{array}{cl}
L_{e, \kappa}\left(\left[\frac{\partial u_{h}^{i+1}}{\partial n}\right] \psi_{e}\right) & \kappa \in\left\{K, K^{\prime}\right\} \\
0 & \text { in } \Omega \backslash\left(K \cup K^{\prime}\right)
\end{array}\right.
$$

where $\psi_{e}$ is the edge-bubble function, $K^{\prime}$ denotes the other element of $\mathcal{T}_{h}$ that share $e$ with $K$ (the operator $L_{e, K}$ was introduced above Proposition 4.2).

Using Cauchy-Schwarz inequality, (2.1), (4.3) and (4.4) we derive

$$
\begin{gathered}
h_{e}^{1 / 2}\left\|\left[\frac{\partial u_{h}^{i+1}}{\partial n}\right]\right\|_{L^{2}(e)}^{2} \leq\left(1+\lambda C\|f\|_{-1, \Omega}^{2 p}\right)\left\|u-u_{h}^{i+1}\right\|_{1, K \cup K^{\prime}}\left\|v_{e}\right\|_{L^{2}(e)} \\
+h_{e}\left\|f-f_{h}\right\|_{L^{2}\left(K \cup K^{\prime}\right)}\left\|v_{e}\right\|_{L^{2}(e)}+h_{e}\left\|f_{h}+\Delta u_{h}^{i+1}-\lambda\left|u_{h}^{i}\right|^{2 p} u_{h}^{i+1}-\alpha\left(u_{h}^{i+1}-u_{h}^{i}\right)\right\|_{L^{2}\left(K \cup K^{\prime}\right)}\left\|v_{e}\right\|_{L^{2}(e)} \\
+C^{\prime}\left(e t a_{K, \beta}^{(L)}+\eta_{K^{\prime}, i}^{(L)}\right)\left\|v_{e}\right\|_{L^{2}(e)}
\end{gathered}
$$

Collecting the two bounds above leads to the following estimation

$$
\eta_{K, i}^{(D)} \leq C \sum_{\kappa \subset \omega_{K}}\left(\left\|u-u_{h}^{i+1}\right\|_{1, \kappa}+\eta_{\kappa, i}^{(L)}+h_{\kappa}\left\|f-f_{h}\right\|_{L^{2}(\kappa)}\right)
$$

These estimates of the local linearization and discretization indicators are fully optimal.

4.2. Algorithm (1.6). The same calculation is followed as before but in (4.6) and (4.7) we have

$\alpha \sum_{K \in \mathcal{T}_{h}} \int_{K} \nabla\left(u_{h}^{i+1}-u_{h}^{i}\right) \nabla v$ instead of $\alpha \sum_{K \in \mathcal{T}_{h}} \int_{K}\left(u_{h}^{i+1}-u_{h}^{i}\right) v$. We are led to define the modified discretization error indicator $\bar{\eta}_{K, i}^{(D)}$ by

$$
\begin{aligned}
\left(\bar{\eta}_{K, i}^{(D)}\right)^{2}=h_{K}^{2}\left\|f_{h}+\Delta u_{h}^{i+1}-\lambda\left|u_{h}^{i}\right|^{2 p} u_{h}^{i+1}+\alpha \Delta\left(u_{h}^{i+1}-u_{h}^{i}\right)\right\|_{L^{2}(K)}^{2} & \\
& +\sum_{e \in \mathcal{E}(K)} h_{e}\left\|\left[\frac{\partial u_{h}^{i+1}}{\partial n}-\alpha \frac{\partial\left(u_{h}^{i+1}-u_{h}^{i}\right)}{\partial n}\right]\right\|_{L^{2}(e)}^{2} .
\end{aligned}
$$

The rest of the calculation is similar. We skip the proofs since they are exactly the same as for Theorems 4.3 and 4.4 .

Theorem 4.5. Upper bound. Let $u_{h}^{i+1}$ and $u$ be the solution of the iterative problem (1.6) and the exact problem (2.2) respectively. We have the following a posteriori error estimate

$$
\left|u_{h}^{i+1}-u\right|_{1, \Omega} \leq C\left(\left(\sum_{K \in \mathcal{T}_{h}}\left(\left(\bar{\eta}_{K, i}^{(D)}\right)^{2}+h_{K}^{2}\left\|f-f_{h}\right\|_{L^{2}(K)}^{2}\right)\right)^{1 / 2}+\left(\sum_{K \in \mathcal{T}_{h}}\left(\eta_{K, i}^{(L)}\right)^{2}\right)^{1 / 2}\right) .
$$

Theorem 4.6. Lower bound. For each $K \in \mathcal{T}_{h}$, there holds

$$
\begin{aligned}
& \eta_{K, i}^{(L)} \leq\left\|u_{h}^{i}-u\right\|_{1, K}+\left\|u_{h}^{i+1}-u\right\|_{1, K}, \\
& \bar{\eta}_{K, i}^{(D)} \leq C \sum_{\kappa \subset \omega_{K}}\left(\left\|u-u_{h}^{i+1}\right\|_{1, \kappa}+\eta_{\kappa, i}^{(L)}+h_{\kappa}\left\|f-f_{h}\right\|_{L^{2}(\kappa)}\right),
\end{aligned}
$$

where $\omega_{K}$ is the union of the triangles sharing at least one edge with $K$.

\section{NumericAl RESUlts}

In this section, we present numerical experiments for our nonlinear problem. These simulations have been performed using the code FreeFem ++ due to F. Hecht and O. Pironneau, see [6]. For all the numerical investigations and for simplicity, we use the finite element of degree $\ell=1$. 
5.1. A priori estimation. We consider the domain $\Omega=]-1,1[2$, each edge is divided into $N$ equal segments so that $\Omega$ is divided into $N^{2}$ equal squares and finally into $2 N^{2}$ equal traingles. We consider the theoretical solution $u=\mathrm{e}^{-5\left(x^{2}+y^{2}\right)}$.

For the convergence, we use the classical stopping criterion $\operatorname{err}_{L} \leq 10^{-5}$, where $e r r_{L}$ is defined by

$$
\operatorname{err}_{L}=\frac{\left|u_{h}^{i+1}-u_{h}^{i}\right|_{1, \Omega}}{\left|u_{h}^{i+1}\right|_{1, \Omega}} .
$$

We consider $\lambda=10, p=50$ and $N=50$. Table 1 shows the error

$$
\operatorname{Err}=\frac{\left|u_{h}^{i}-u\right|_{1, \Omega}}{|u|_{1, \Omega}}
$$

which describes the convergence of the algorithms (1.5) and (1.6) with respect of $\alpha$. We remark that the algorithm (1.5) converges for $\alpha \geq 21.82$ and the algorithm (1.6) converges for $\alpha \geq 0.77$.

In order to compare our algorithms (1.5) and (1.6) with (1.4), Table 2 shows the convergence for $N=50$

\begin{tabular}{|l|l|l|l|l|l|l|}
\hline$\alpha$ & $\mathbf{0 . 0 1}$ & $\mathbf{0 . 5}$ & $\mathbf{0 . 7 6}$ & $\mathbf{0 . 7 7}$ & $\mathbf{1}$ & $\mathbf{1 0}$ \\
\hline Algo (1.5) & div & $\operatorname{div}$ & $\operatorname{div}$ & $\operatorname{div}$ & $\operatorname{div}$ & $\operatorname{div}$ \\
\hline Algo (1.6) & div & div & div & 0.0581902 & 0.0581908 & 0.0581905 \\
\hline & $\mathbf{7}$ \\
\hline$\alpha$ & $\mathbf{2 0}$ & $\mathbf{2 1 . 8 1}$ & $\mathbf{2 1 . 8 2}$ & $\mathbf{2 2}$ & $\mathbf{5 0}$ & $\mathbf{1 0 0}$ \\
\hline Algo (1.5) & div & div & 0.0581906 & 0.0581904 & 0.0581897 & 0.0581886 \\
\hline Algo (1.6) & 0.0581906 & 0.0581907 & 0.0581907 & 0.0581907 & 0.0581919 & 0.0581973 \\
\hline
\end{tabular}

TABLE 1. Convergence of algorithms (1.5) and (1.6) with respect of $\alpha$.

and a fixed $\alpha=22$ in our algorithms. In fact, for big values of $\lambda$ and $p$, the algorithm (1.4) diverges. We mention that for $\lambda$ and $p$ where (1.5) and (1.6) diverge, we must take a bigger values of $\alpha$ to obtain the convergence. Figure 1 shows in logarithmic scale the error Err with respect to $h$ (algorithm 1.5 in the left

\begin{tabular}{|l|l|l|l|l|l|}
\hline & $\lambda=1$ & $\lambda=2$ & $\lambda=5$ & $\lambda=5$ & $\lambda=10$ \\
& $p=1$ & $p=10$ & $p=10$ & $p=50$ & $p=10$ \\
\hline Algo (1.4) & 0.0581392 & 0.0580725 & $\operatorname{div}$ & $\operatorname{div}$ & $\operatorname{div}$ \\
\hline Algo (1.5) & 0.0580467 & 0.058072 & 0.0581392 & 0.0581284 & 0.0582106 \\
\hline Algo (1.6) & 0.0580458 & 0.0580717 & 0.0581399 & 0.0581292 & 0.0582111 \\
\hline & \multicolumn{5}{|l|}{} \\
\hline & $\lambda=10$ & $\lambda=10$ & $\lambda=50$ & $\lambda=100$ & $\lambda=100$ \\
& $p=50$ & $p=100$ & $p=50$ & $p=50$ & $p=100$ \\
\hline Algo (1.4) & div & div & div & div & div \\
\hline Algo (1.5) & 0.0581904 & 0.0581759 & div & div & div \\
\hline Algo (1.6) & 0.0581907 & 0.0581764 & 0.0582894 & 0.0583124 & 0.0582956 \\
\hline
\end{tabular}

TABLE 2. Comparison of the convergence of algorithms (1.5), (1.6) (for $\alpha=22$ ) with (1.4).

and algorithm 1.6 in the right). The slope of the error corresponding to (1.5) and (1.6) are respectively 0.92 and 0.96 , which validates Theorem 3.2. 

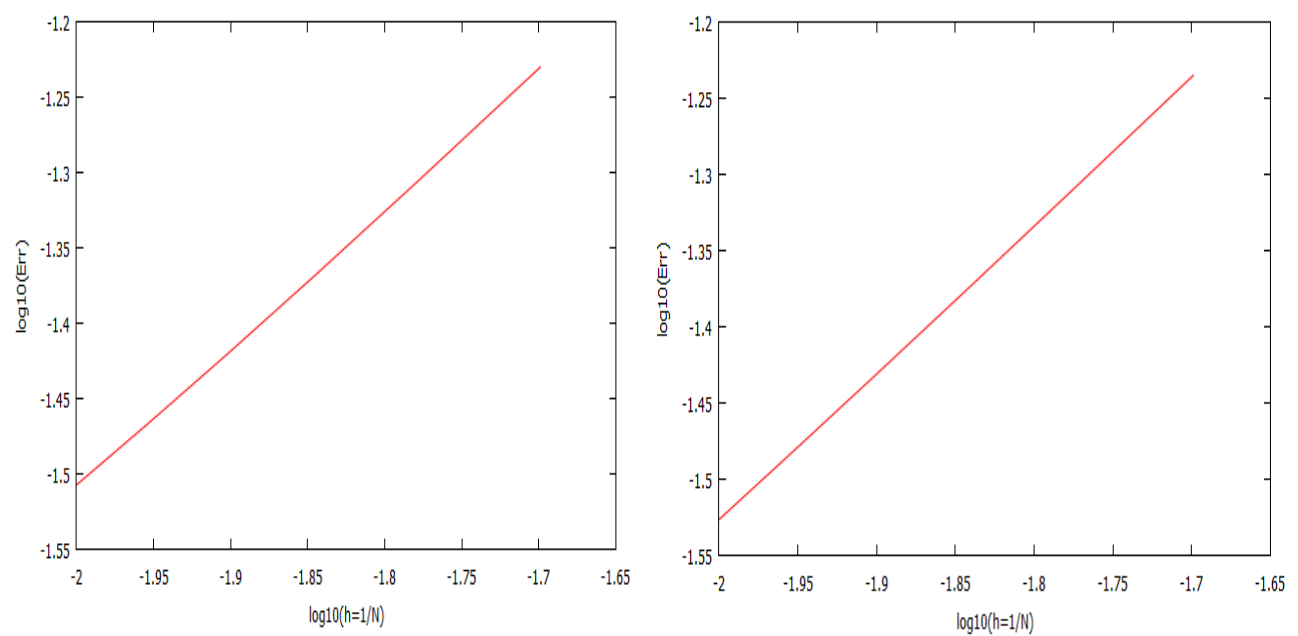

FiguRE 1. A priori error with respect of $h=1 / N$ : left (algo 1.5) and right (algo 1.6).

5.2. A posteriori analysis. In this section, we test our a posteriori error estimates on our model problem. We consider the same domain $\Omega$ with the theoretical solution now given by $u=\mathrm{e}^{-100\left(x^{2}+y^{2}\right)}$. and we choose $\lambda=10$ and $p=50$.

In [2] and for the adaptive strategy, we define the global indicators (introduced in [5]):

$$
\eta_{i}^{(D)}=\left(\sum_{K \in \mathcal{T}_{h}}\left(\eta_{K, i}^{(D)}\right)^{2}\right)^{1 / 2} \quad \text { and } \quad \eta_{i}^{(L)}=\left(\sum_{K \in \mathcal{T}_{h}}\left(\eta_{K, i}^{(L)}\right)^{2}\right)^{1 / 2},
$$

and we introduce two kinds of stopping criteria :

$$
\eta_{i}^{(L)} \leq 10^{-5} \quad \text { Classical stopping criterion },
$$

and

$$
\eta_{i}^{(L)} \leq \gamma \eta_{i}^{(D)} \quad \text { New stopping criterion }
$$

where $\gamma$ is a parameter which balances the discretization and linearization errors. We studied in [2] the comparison between these two types of stopping criterion and we showed the efficiency of the new one which is considered in this paper with $\gamma=0.001$.

For our numerical investigations, we follow the algorithm described in [2]. The evolution of the meshes with the new stopping criterion looks like the figures 3 and 4 in [2]. We note that for $\lambda=10$ and $p=50$, the algorithm (1.4) diverges.

Figure 2 gives a comparison in logarithmic scale of the error between the uniform and adaptive methods using the algorithms (1.5) and (1.6) with respect of the number of vertices. We can easily see that the algorithms (1.5) and (1.6) give comparable results but the adaptive method is more powerful than the uniform one.

Table 3 shows comparisons, for approximatively the same precision, of the CPU time between the algorithm (1.5) and (1.6) with respect of $\alpha$. We remark that algorithm (1.5) is faster than (1.6).

In order to have an idea of the constant on the upper bound in theorem 4.3, Table 4 shows the repartition of the error Err and the sum of the indicators

$$
\operatorname{err}_{I}=\frac{\left(\left(\eta_{i}^{(D)}\right)^{2}+\left(\eta_{i}^{(L)}\right)^{2}\right)^{1 / 2}}{|u|_{1, \Omega}} \simeq \frac{\eta_{i}^{(D)}}{|u|_{1, \Omega}}
$$

during the refinement level and after the convergence on each one. Even if the errors regularly decrease (for instance from 1 to 0.14 for $\operatorname{err}_{I}$ ) with respect to the number of adaptive refinement levels which is 


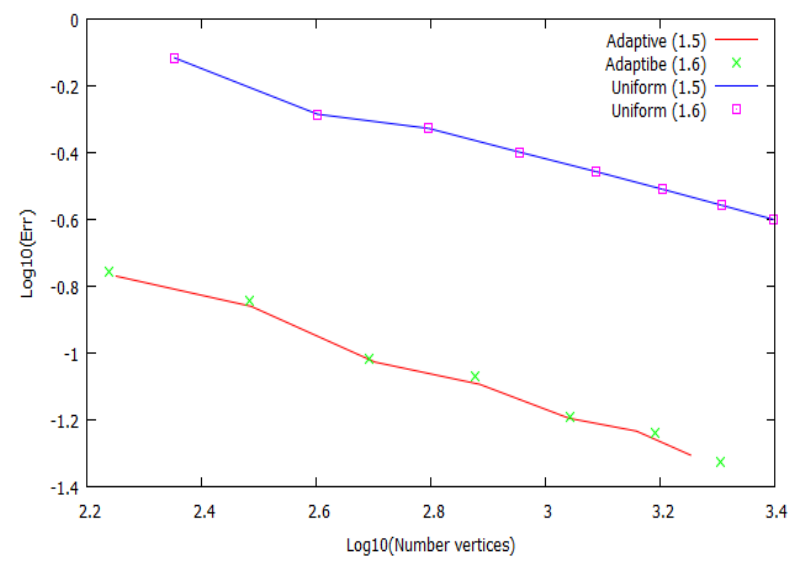

FiguRE 2. Error versus number of vertices in logarithmic scale for adaptive and uniform methods with algorithms (1.5) and (1.6).

\begin{tabular}{|l|l|l|l|l|}
\hline$\alpha$ & $\mathbf{2 2}$ & $\mathbf{3 0}$ & $\mathbf{4 0}$ & $\mathbf{5 0}$ \\
\hline Algo (1.5) & time=5.186 s & time=4.952 $\mathrm{s}$ & time=5.505 $\mathrm{s}$ & time=5.51 \\
& error $=0.0487858$ & error=0.0484306 & error=0.048256 & error=0.0478781 \\
\hline Algo (1.6) & $\begin{array}{l}\text { time=56.625 } \mathrm{s} \\
\text { error }=0.0475732\end{array}$ & $\begin{array}{l}\text { time=59.499 } \\
\text { error=0.0486143 }\end{array}$ & $\begin{array}{l}\text { time=66.231 } \\
\text { error=0.0469877 }\end{array}$ & $\begin{array}{l}\text { time=61.268 } \mathrm{s} \\
\text { error=0.0494427 }\end{array}$ \\
\hline
\end{tabular}

TABLE 3. Comparison of the precision and the CPU time between the algorithm (1.5) and (1.6) with respect of $\alpha$.

\begin{tabular}{llll}
\hline Itn & Err & err $_{I}$ & $C=\frac{e r r_{I}}{E r r}$ \\
\hline 1 & 0.426417 & 1.31249 & 3.07796 \\
2 & 0.169927 & 0.524469 & 3.08644 \\
3 & 0.138091 & 0.372655 & 2.69862 \\
4 & 0.093948 & 0.278664 & 2.96615 \\
5 & 0.0806374 & 0.222064 & 2.75386 \\
6 & 0.063787 & 0.186245 & 2.91979 \\
7 & 0.0583991 & 0.160167 & 2.74262 \\
8 & 0.049479 & 0.14235 & 2.87698
\end{tabular}

\begin{tabular}{llll}
\hline Itn & Err & err $_{I}$ & $C=\frac{e r r_{I}}{E r r}$ \\
\hline 1 & 0.443195 & 1.29673 & 2.92587 \\
2 & 0.175596 & 0.536429 & 3.05491 \\
3 & 0.143715 & 0.38263 & 2.66242 \\
4 & 0.0959932 & 0.290036 & 3.02142 \\
5 & 0.0848493 & 0.229053 & 2.69953 \\
6 & 0.0643159 & 0.191061 & 2.97066 \\
7 & 0.0577646 & 0.157273 & 2.72265 \\
8 & 0.0474228 & 0.137726 & 2.90422
\end{tabular}

TABLE 4. Repartition of errors and indicators during the refinement levels (Itn) : Left (algorithm (1.5)) and right (algorithm (1.6)).

consistent with adapted mesh method, the constant remains stable and can be approximated by 2.85 . 


\section{REFERENCES}

[1] Adams R.A., Sobolev Spaces, Acadamic Press, INC, 1978.

[2] Bernardi C., Dakroub J., Mansour G., Sayah T., A posteriori analysis of iterative algorithms for a nonlinear problem, to appear in Journal of Scientific Computing, (2015).

[3] Chaillou A.-L., Suri M., Computable error estimators for the approximation of nonlinear problems by linearized models, Computable Methods in Applied Mechanics and Engineering 196, 210-224, (2006).

[4] Chaillou A.-L., Suri M., A posteriori estimation of the linearization error for strongly monotone nonlinear operators, Computable Methods in Applied Mechanics and Engineering 205, 72-87, (2007).

[5] El Alaoui L., Ern A., Vohralík M., Guaranteed and robust a posteriori error estimate and balancing discretization and linearization errore for monotone nonlinear problems, Computable Methods in Applied Mechanics and Engineering 200, 2782-2795, (2011).

[6] Hecht F., New development in FreeFem++, Journal of Numerical Mathematics 20, 251-266, (2012).

[7] Verfürth R., A Posteriori Error Estimation Techniques For Finite Element Methods, Numerical Mathematics And Scientific Computation, Oxford, 2013. 